\title{
Binding energies of excitons in II-VI compound-semiconductor based quantum well structures
}

\author{
R. T. Senger ${ }^{*}, 1,2$ and K. K. Bajaj ${ }^{3}$ \\ ${ }^{1}$ TÜBİTAK - UEKAE, P.K. 74, 41470 Gebze, Kocaeli, Turkey \\ 2 Department of Physics, Bilkent University, 06800 Bilkent, Ankara, Turkey \\ ${ }^{3}$ Department of Physics, Emory University, Atlanta GA 30322, USA
}

Received 27 February 2004, revised 19 April 2004, accepted 21 April 2004

Published online 3 June 2004

\section{PACS 71.35.Cc, 78.67.De, 78.66.Hf}

We present a brief description of the calculation of the variation of the binding energy of the heavy-hole exciton as a function of well width in quantum well structures composed of II-VI compound semiconductors including the effects of exciton-optical phonon interaction as formulated by Pollmann and Büttner [J. Pollmann and H. Büttner, Phys. Rev. B 16, 4480 (1977)], and of particle masses and dielectric mismatches between the well and the barrier layers. We compare the results of our calculations with the available experimental data in $\mathrm{ZnSe} / \mathrm{MgS}$, $\mathrm{ZnSe} / \mathrm{Mg}_{0.15} \mathrm{Zn}_{0.85} \mathrm{Se}$, and $\mathrm{ZnS} / \mathrm{Mg}_{0.19} \mathrm{Zn}_{0.81} \mathrm{~S}$ quantum well structures and find a good agreement.

\section{Introduction}

There has been an enormous interest in the study of the structural, electronic and optical properties of quantum well structures based on II-VI compounds during the past fifteen years. This interest has been motivated by a desire to understand their fundamental properties as well as by the prospectus of their potential applications in opto-electronic devices operating in the highly desirable blue-green region of the electromagnetic spectrum. A variety of quantum well structures such as $\mathrm{ZnSe} / \mathrm{MgS}, \mathrm{ZnCdSe} / \mathrm{ZnSe}$, $\mathrm{ZnO} / \mathrm{ZnMgO}, \mathrm{ZnSe} / \mathrm{ZnMgSe}$, and $\mathrm{ZnS} / \mathrm{ZnMgS}$, etc. have been fabricated using epitaxial crystal growth techniques such as molecular beam epitaxy (MBE) and metal-organic chemical vapor deposition (MOCVD), and their electronic properties have been investigated using several optical characterization techniques. The binding energies of excitons in these quantum well structures are considerably larger than in those based on common III-V semiconductors and hence excitonic recombination can play an important role in lasing emission processes even at room temperature. Excitonic transitions have much higher oscillator strengths and more peaked density of states than those corresponding to free carriers thus leading to improved laser characteristics.

Recently, the variations of the binding energies of exciton as a function of well width have been measured in $\mathrm{ZnSe} / \mathrm{MgS}$ [1], $\mathrm{ZnSe} / \mathrm{ZnMgSe}$ [2], and $\mathrm{ZnS} / \mathrm{ZnMgS}$ [3] quantum well structures. These are highly ionic materials in which the excitons interact rather strongly with the longitudinal optical (LO) phonon modes of the heterostructure. Zheng and Matsuura [4] have calculated the variations of the exciton binding energies as a function of well width in ionic quantum well structures taking into account the interaction of the exciton with confined LO phonons, interface phonons, and half-space phonons. Recently, we have shown [5] that the values of the exciton binding energies calculated using an effective

\footnotetext{
"Corresponding author: e-mail: senger@fen.bilkent.edu.tr
} 
interaction potential between the electron and the hole as derived by Pollmann and Büttner [6] using exciton-bulk LO phonon interaction, agree very well with those calculated by Zheng and Matsuura [4].

In this paper we present the results of our calculations of the variation of the binding energies of excitons as a function of well width in $\mathrm{ZnSe} / \mathrm{MgS}$, $\mathrm{ZnSe} / \mathrm{ZnMgSe}$, and $\mathrm{ZnS} / \mathrm{ZnMgS}$ quantum well structures using an effective interaction Hamiltonian as derived by Pollmann and Büttner (PB) [6]. In our calculations we have included the effects of mismatches between the dielectric constants and the particle masses of the well and the barrier regions whenever the values of such physical parameters are reliably known for the barrier layers. For the sake of comparison we have also calculated the variation of the exciton binding energies as a function of well size using statically screened Coulomb potential between the electron and the hole, and the polaronic values of the electron and the hole masses. We compare the results of our calculations with the available experimental data in the above mentioned quantum well structures and find a very good agreement when the electron-hole interaction is described by PB potential. The use of statically screened potential between the electron and the hole always leads to significantly lower values of the exciton binding energies. Similar results have been found in other systems such as $\mathrm{ZnO} / \mathrm{ZnMgO}$ and $\mathrm{ZnCdSe} / \mathrm{ZnSe}$ quantum well structures [5].

\section{Theory}

The details of our calculations of the binding energies of excitons in ionic quantum well structures have already been reported elsewhere [5]. The same formalism was used for the study of excitonic properties in polar quantum dots and satisfactory results were obtained [7, 8]. Here we provide a very brief description of our calculations, and the results of the application of our formalism to the above mentioned $\mathrm{ZnSe}$ and $\mathrm{ZnS}$ based quantum wells for the sake of completeness. For the discussion of the PB Hamiltonian and the details of our calculations the reader is referred to [5].

We consider an electron-hole pair confined in a quantum well with band offsets $V_{e}$ and $V_{h}$, and in interaction with the longitudinal optical phonon modes. The values of the particle masses are treated as position dependent being different in the well and the barrier regions of the quantum well. The anisotropic hole mass along the direction of the growth of the quantum well and in the transverse plane is determined in terms of the well-known Luttinger band parameters [9]. The interaction of an electron and a hole is modified in a quantum well due to dielectric discontinuities at the interfaces. To account for the effects of dielectric confinement on the electron-hole Coulomb interaction we use an effective potential term, $V_{\mathrm{KT}}$, as derived by Kumagai and Takagahara [10] using the image charge method. Pollmann and Büttner were able to describe quantitatively the effect of exciton-phonon coupling through an effective interaction potential between the electron and the hole and a self-energy term [6]. We have adopted that approach to the case of quantum well confinement geometry. The explicit forms of all the terms of the effective Hamiltonian can be found in Ref. [5]. To calculate the ground state energy of the heavy-hole exciton we minimize the expectation value of the Hamiltonian calculated with a trial wave function having two variational parameters [5]. The exciton binding energy is then determined with reference to the sum of the confinement subband energies and the polaronic self-energies of an uncorrelated electron and hole configuration in the quantum well. It should be noted that polaronic mass renormalizations are not required in the PB formulation [6].

For the demonstration of the importance of proper accounting for the exciton-phonon interaction in highly ionic quantum well structures we have also calculated the exciton binding energies using a simpler model which treats the exciton as composed of an electron-polaron and a hole-polaron with polaronic particle masses and interacting through statically screened Coulomb potential. The mass-mismatch and dielectric mismatch effects are also treated the same way in this model.

\section{Results and conclusions}

We have calculated the variation of the binding energy $\left(E_{B}\right)$ of a heavy-hole exciton as a function of well width $(L)$ in several quantum well structures composed of II-VI semiconductors. The values of the various physical parameters used in our calculations are given in Table 1. 
Table 1 Values of the various physical parameters used in our calculations. All mass values are without polaronic corrections and are expressed in units of free electron mass $\left(m_{0}\right)$. Conduction and valance band offsets, $V_{e}$ and $V_{h}$, and LO phonon energy $\hbar \omega_{\mathrm{LO}}$ are given in meV. Static $\left(\varepsilon_{0}\right)$ and optical $\left(\varepsilon_{\infty}\right)$ dielectric constants are those of the well material. The Luttinger parameters $\gamma_{1}=2.45$ and $\gamma_{2}=0.61$ are used for the anisotropic heavy-hole mass values of $\mathrm{ZnSe}[11]$.

\begin{tabular}{|c|c|c|c|c|c|c|c|c|}
\hline well/barrier & $m_{e}$ & $m_{h}^{z}$ & $m_{h}^{\| \prime}$ & $\varepsilon_{0}$ & $\varepsilon_{\infty}$ & $V_{e}$ & $V_{h}$ & $\hbar \omega_{\mathrm{LO}}$ \\
\hline a) $\mathrm{ZnSe} / \mathrm{MgS}$ & 0.14 & 0.81 & 0.33 & 8.8 & 5.73 & 1384 & 593 & 31.7 \\
\hline b) $\mathrm{ZnSe} / \mathrm{Mg}_{0.15} \mathrm{Zn}_{0.85} \mathrm{Se}$ & 0.14 & 0.81 & 0.33 & 8.8 & 5.73 & 134 & 58 & 31.7 \\
\hline${ }^{\mathrm{c})} \mathrm{ZnS} / \mathrm{Mg}_{0.19} \mathrm{Zn}_{0.81} \mathrm{~S}$ & 0.20 & 0.73 & 0.73 & 8.9 & 5.10 & 87 & 87 & 44.0 \\
\hline
\end{tabular}

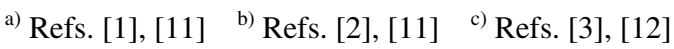

In Fig. 1 we display the variation of the exciton binding energy as a function of well width in $\mathrm{ZnSe} / \mathrm{MgS}$ quantum well structures. The solid curve represents the results of our calculation using PB potential. The effects of mismatches of the particle masses and dielectric constants between the well and the barrier layers are not included as the values of these parameters are not known in the barrier layers. The dashed curve represents the results of our calculation using statically screened Coulomb potential. Solid squares represent the measured values of $E_{B}$ by Urbaszek et al. [1]. They performed magnetoabsorption measurements at $4 \mathrm{~K}$ and determined the values of $E_{B}$ from the zero-field extrapolated values of the energy differences between $1 s$ and $2 s$ states. It is clear from Fig. 1 that the values of $E_{B}$ obtained using PB potential are considerably larger than those calculated using the statically screened potential especially for narrow wells. The measured values of $E_{B}$ agree rather well with those calculated using PB potential, thus emphasizing the importance of exciton-phonon interaction in this quantum well structure.

In Fig. 2 we display the variation of the exciton binding energy as a function of well width in a $\mathrm{ZnSe} / \mathrm{Mg}_{0.15} \mathrm{Zn}_{0.85}$ Se quantum well structure using the values of the various physical parameters given in Table 1. The solid curve and the dashed curve have the same meaning as in Fig. 1. The solid square represents the value of $E_{B}$ measured by Puls et al. [2] in a quantum well of width of $45 \AA$. Though they

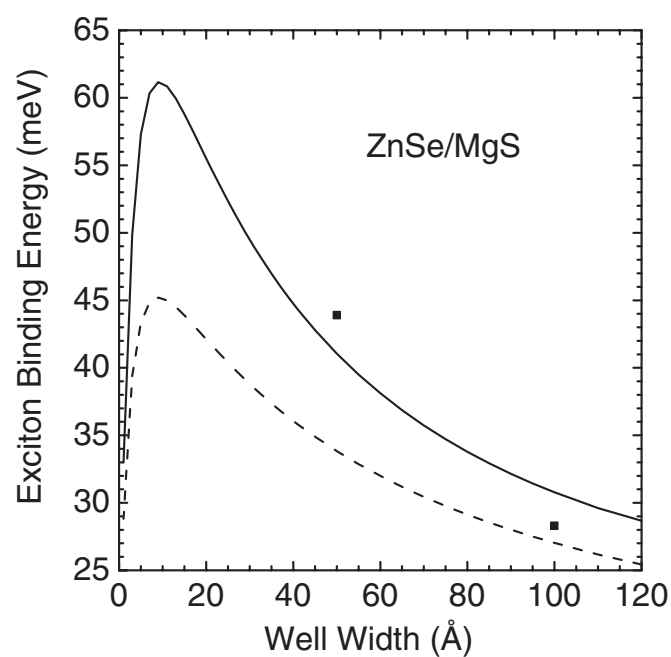

Fig. 1 Variation of exciton binding energy as a function of well width in $\mathrm{ZnSe} / \mathrm{MgS}$ quantum wells. The solid and dashed curves are obtained using PB and statically screened Coulomb potentials, respectively. The symbols refer to the experimental data by Urbaszek et al. [1].

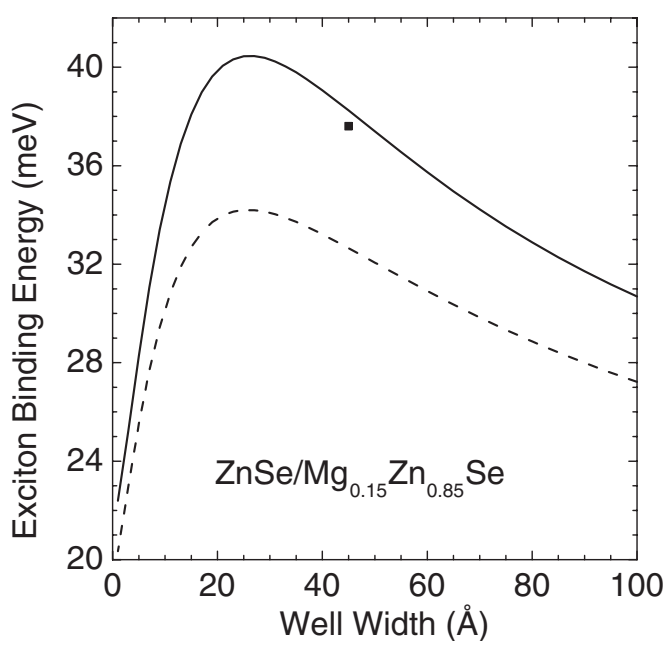

Fig. 2 Variation of exciton binding energy as a function of well width in $\mathrm{ZnSe} / \mathrm{Mg}_{0.15} \mathrm{Zn}_{0.85}$ Se quantum wells. The solid and dashed curves have the same meaning as in Fig. 1. The symbol is an experimental measurement by Puls et al. [2]. 


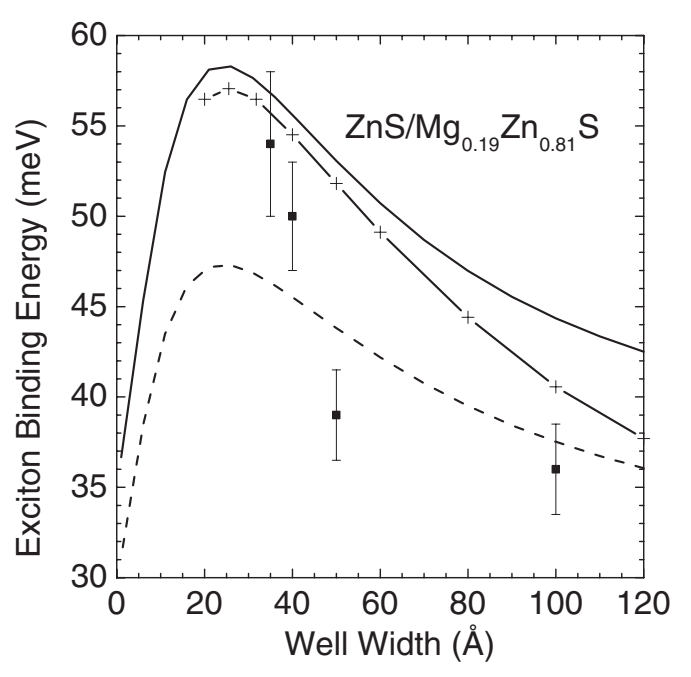

Fig. 3 Variation of exciton binding energy as a function of well width in $\mathrm{ZnS} / \mathrm{Mg}_{0.19} \mathrm{Zn}_{0.81} \mathrm{~S}$ quantum wells. The solid and dashed curves have the same meaning as in Fig. 1. The experimental data by Urbaszek et al. [3] are represented by symbols. The solid curve with (+) symbols represents the results of a theoretical calculation with the model of Peyla et al. [13].

grew and studied several quantum well structures with varying values of $\mathrm{Mg}$ compositions and well widths, this particular sample was investigated in considerable detail using magneto-PLE measurements at $5 \mathrm{~K}$. They determine a value of $37.6 \mathrm{meV}$ for the binding energy of a heavy-hole exciton which agrees remarkably well with the value we calculate using PB potential. Again the use of statically screened Coulomb potential significantly underestimates the values of $E_{B}$. The reasons for the choice of physical parameters of $\mathrm{ZnSe}$ we have used in our calculations have been discussed in some detail by Nardis et al. [11].

In Fig. 3 we plot the variation of the exciton binding energy as a function of well width in $\mathrm{ZnS} / \mathrm{Mg}_{0.19} \mathrm{Zn}_{0.81} \mathrm{~S}$ quantum well structure. The value of the hole mass in $\mathrm{ZnS}$ was obtained by equating the calculated value of the exciton binding energy using PB potential with the measured value (36.0 meV) in bulk $\mathrm{ZnS}$. The solid curve and the dashed curve have the same meaning as in Fig. 1 and Fig. 2. The solid squares represent the values of the exciton binding energy measured by Urbaszek et al. [3]. The values of the well widths ranged from 35 to $100 \AA$. The values of the exciton binding energies for narrow wells agree rather well with those calculated using PB potential. However, the values of the exciton binding energies in two broader wells are even less than those calculated using statically screened Coulomb potential. There is a dramatic drop in the value of $E_{B}$ as one goes from $40 \AA$ to $50 \AA$ wide well. The measured value of exciton binding energy in the $100 \AA$ wide well is equal to the bulk value $36 \mathrm{meV}$ although there should be significant confinement effects. The reason for this strange behavior is not clear. It should be pointed out that Urbaszak et al. [3] do not measure the values of $E_{B}$ directly. They determine the energy difference between the $1 s$ and $2 s$ exciton states and then derive the value of $E_{B}$ using a calculation which does not include the effect of exciton-phonon interaction. This procedure is likely to introduce some error in the determination of $E_{B}$. However they use the same procedure to determine the values of $E_{B}$ in narrow wells where these values seem very reasonable. Clearly more work is needed in this heterostructure before one can meaningfully compare theory with experiment. Urbaszek et al. [3] compare their experimental data with the results of a calculation by Peyla et al. [13], which we also include in Fig. 3 as the solid curve with symbols. They numerically solve the Schrödinger equation of the exciton treating the heavy-hole mass as a variational parameter. Exciton phonon interaction is not included in their treatment. It is seen that the values of binding energies calculated with PB model are comparable to those of Peyla et al. in narrow wells; however the discrepancy between the two theories increases towards the bulk limit. It should be pointed out that the values of the physical parameters used by Urbaszek et al. [3] in their calculations are quite different from those used by us. For instance, the value of the mass of the conduction electron in $\mathrm{ZnS}$ they use $\left(0.34 \mathrm{~m}_{0}\right)$ is considerably larger than the one $\left(0.20 m_{0}\right)$ used by us. The latter value was determined by Imanaka and Miura [12] using cyclotron resonance technique which is known to give very accurate values of the band 
masses. In addition Urbaszek et al. [3] use hole mass as an adjustable parameter and choose $m_{h}=0.387 m_{0}$. We have calculated the values of $E_{B}$ using the set of parameters suggested by Urbaszek et al. [3] in the PB model. We find that the values of the binding energies thus obtained are larger by about $3 \mathrm{meV}$. There are no adjustable parameters in our calculations. We should mention that the effects of the valence band mixing are not included in our calculations as our main objective is to investigate the contribution of the exciton-phonon interaction to the exciton binding energies in II-VI based quantum well structures.

In summary, we have briefly described the calculation of the variation of the binding energy of a heavy-hole exciton as a function of well width in quantum well structures composed of II-VI compound semiconductors including the effects of exciton-optical phonon interaction as formulated by Pollmann and Büttner and the particle masses and dielectric mismatches between the well and the barrier materials. We compare the results of our calculations with the available experimental data in several quantum well structures and find in general a good agreement, demonstrating the importance of exciton-optical phonon interactions in these systems, for proper calculations of exciton binding energies.

\section{References}

[1] B. Urbaszek, A. Balocchi, C. Bradford, C. Morhain, C. B. O’Donnel, K. A. Prior, and B. C. Cavenett, Appl. Phys. Lett. 77, 3755 (2000).

[2] J. Puls, M. Rabe, A. Siarkos, and F. Henneberger, Phys. Rev. B 57, 14749 (1998).

[3] B. Urbaszek, C. M. Townsley, X. Tang, C. Morhain, A. Balocchi, K. A. Prior, R. J. Nicholas, and B. C. Cavenett, Phys. Rev. B 64, 155321 (2001).

[4] R. Zheng and M. Matsuura, Phys. Rev. B 58, 10769 (1998).

[5] R. T. Senger and K. K. Bajaj, Phys. Rev. B 68, 205314 (2003).

[6] J. Pollmann and H. Büttner, Phys. Rev. B 16, 4480 (1977).

[7] R. T. Senger and K. K. Bajaj, phys. stat. sol. (b) 236, 82 (2003).

[8] R. T. Senger and K. K. Bajaj, Phys. Rev. B 68, 045313 (2003).

[9] A. Baldareschi and N. O. Lipari, Phys. Rev. B 3, 439 (1971).

[10] M. Kumagai and T. Takagahara, Phys. Rev. B 40, 12359 (1989).

[11] A. De Nardis, V. Pellegrini, R. Colombelli, F. Beltram, L. Vanzetti, A. Franciosi, I. N. Krivorotov and K. K. Bajaj, Phys. Rev. B 61, 1700 (2000).

[12] Y. Imanaka and N. Miura, Phys. Rev. B 50, 14065 (1994).

[13] P. Peyla, R. Romestain, Y. Merle d'Aubingé, G. Fishman, A. Wasiela, and H. Mariette, Phys. Rev. B 52, 12026 (1995). 